\title{
Nutrição aplicada à Atividade Motora
}

\author{
Antonio Herbert LANCHA J UNIOR* \\ *Escola de Educa- \\ ção Física e Esporte, \\ Universidade de São \\ Paulo.
}

\section{Resumo}

A Nutrição aplicada a atividade motora se divide em quatro áreas do movimento humano, a saber: esporte, educação física, dança, recreação/lazer. Essa definição conceitual diferencia a população alvo da intervenção nutricional. 0 organismo humano sempre apresentou o movimento como parte de sua atividade cotidiana e selecionou evolutivamente os organismos mais econômicos. Em contrapartida por conta de demandas, sociais, financeiras dentre outras a vida moderna impôs o sedentarismo como padrão de comportamento motor que aliado ao padrão genético de economia resultaram nas doenças modernas como obesidade, diabetes, etc. Assim a sociedade institucionalizou o movimento humano criando manifestações distintas descritas acima e suas necessidades específicas passaram a ser de interesse acadêmico/cientifico. Nutricionalmente os estudos se concentram no balanço energético, na necessidade de carboidratos, proteinas, lipídios assim como dos micronutrientes e outros compostos biologicamente ativos. Estes estudos definem estas substancias sob critérios de essencialidade ou efeito ergogênico superior a capacidade fisiológica. 0 primeiro determina mudanças nas necessidades nutricionais e o segundo substâncias consideradas ilícitas. No presente momento grande parte da comunidade cientifica dedicada à nutrição aplicada à atividade motora, dirige sua vocação na tentativa de descobrir as necessidades específicas provocadas pela pratica regular da atividade motora permitindo seu exercício regular para que a mesma propicie os benefícios na manutenção da saúde de forma plena nas quatro áreas descritas acima.

UnITERMOS: Nutrição; Esporte; Educação física.

\section{Nutrição aplicada a Atividade Motora}

Historicamente o nosso padrão genético se assemelha ao do hominídeo que ocupou a terra a milhōes de anos atrás. Esse padrão evoluiu em um ambiente hostil onde a disponibilidade de alimentos ocorria por seis meses do ano restando os outros seis (outono e inverno) com baixa ou nenhuma oferta alimentar. Diferentemente de outras espécies que conseguiam reduzir sua necessidade energética com manobras metabólicas elaboradas como a hibernação, o hominídeo mantinha o padrão metabólico de sono e vigília e com isso a necessidade de energia elevada. Foram selecionados então os seres mais eficientes em estocar energia, ou seja, os mais aptos a engordar.

Andando rapidamente na linha do tempo, o homem contemporâneo passou a apresentar um comportamento diferenciado após a o período cibernético. $\mathrm{O}$ computador que historicamente surgiu para trabalhar pelo homem elevando o seu tempo livre, passou a ser usado no tempo livre elevando a produtividade. Com isso a atividade motora no dia a dia ficou prejudicada e o gasto energético também. Colocando essas variáveis juntas, o padrão genético econômico do hominídeo aliado ao gasto calórico reduzido do homem moderno as consequências são a pandemia de obesidade, o diabetes e etc.

A atividade motora foi então institucionalizada de diferentes formas na nossa cultura recente. Ao discutirmos nutrição aplicada à atividade motora, devemos inicialmente descrever as formas diferentes de manifestação do movimento humano. Para tanto classificaremos essas manifestaçōes em quatro situaçōes distintas como descrito por LANCHA JUNIOR e RIANI (2011): 1) Esporte: prática classificada pelas características competitivas com regras definidas, em que seus praticantes são classificados com atletas. $\mathrm{O}$ profissional que atua nesta área apresenta Bacharelado em Esporte e tem sua formação destacada para o desempenho do praticante em suas diversas áreas do conhecimento (biodinâmico psicodinâmico e sociodinâmico). $\mathrm{O}$ atleta tem como diferencial do 
indivíduo comum a prática esportiva realizada no seu limite de competência fisiológica e biomecânica. $\mathrm{O}$ atleta faz do seu desempenho esportivo sua forma de atuação profissional, ou seja, sua profissão, e desempenha aquela modalidade no seu limite pessoal. Então, ele se destaca dos demais indivíduos por apresentar resultados no limite superior ao padrão de normalidade populacional. Com isso temos os atletas respondendo pelas performances extremas dos humanos, as quais são avaliadas periodicamente em competições. As competiçôes, em que os critérios de julgamento da modalidade são padronizados e as condiçôes ambientais e de equipamentos devem ser reprodutivas, servem para avaliar, na linha do tempo, se outro atleta desempenhou, na mesma modalidade, melhor resultado. Este melhor resultado é registrado como "record". Assim, a definição de atleta tem fim em si próprio e nãopode ser confundida com indivíduos fisicamente ativos. 2) Dança: prática classificada pela expressão corporal na qual seus praticantes desempenham, dentro do seu limite de competência, a melhor expressão do tema proposto. Seus praticantes são os dançarinos ou ainda, como herança do balé, bailarinos, o que consideramos como termo genérico, equivocado. A dança é por vezes competitiva, trazendo as características do esporte para si. Desse modo, as regras devem ser definidas e sua avaliação deve seguir, dentro do possível, padrões específicos para garantir sua reprodutibilidade. Como no esporte, seus praticantes desempenham a dança dentro do seu limite de competência fisiológica e biomecânica e são destacados os que apresentam melhores capacidades físicas, como flexibilidade e força, além de habilidades como saltar, girar e aterrissar, equilibrar etc. aliadas às expressóes artísticas. Podemos distinguir o dançarino do atleta, pois este agrega, ao desempenho, a conotação artística. 3) Recreação e lazer: prática definida como lúdica, com o objetivo de ocupação do tempo livre com atividades corporais. Pode se valer de modalidades esportivas, porém o praticante apresenta seu desempenho dentro da normalidade humana. Em alguns países essa prática é utilizada para avaliar desempenho destacado em crianças e adolescentes, com o objetivo de selecionar talentos nas habilidades esportivas e/ou artísticas. Nesse caso os profissionais que aplicam as tarefas possuem formação capaz de preparar o ambiente e observar o praticante diferenciando-o dos demais. O praticante de atividades de recreação e lazer é caracterizado pela grande maioria da população. Assim, pode haver uma modalidade esportiva com praticantes classificados como atletas e indivíduos se recreando. Um exemplo cada vez mais popular são as corridas de rua, em que o vencedor de uma Maratona, por exemplo, percorre os 42.195 metros em pouco mais de duas horas e o indivíduo comum necessita de duas ou três vezes esse tempo. Este dado enfatiza a atenção a ser dada a este praticante recreativo, pois sua exposição a fatores ambientais como vento, temperatura e umidade relativa do ar o tornará mais vulnerável que o atleta a desidratação, hipertermia e demais ocorrências dessa natureza. 4. Educação física: prática sistemática que se utiliza de testes fisiológicos e metabólicos, além de avaliações de composição corporal, capacidades e habilidades motoras para aferir o estado de saúde do seu praticante ao longo do tempo. O profissional que atua nesta área é o Bacharel em Educação Física e/ ou aquele que faz a licenciatura nesta área, chamado de professor de educação física, o qual apresenta competências de um educador e domina as diversas áreas do conhecimento (biodinâmico, psicodinâmico e sociodinâmico). Na nossa cultura, o profissional de educação física tem por objetivo o desenvolvimento de habilidades, como equilíbrio, coordenação e agilidade, além de capacidades, conhecidas como elementos definidores da aptidão física, que são a força, a resistência aeróbia, a flexibilidade, etc.

Com essas definições podemos abordar a nutrição aplicada à atividade motora com objetivos distintos em cada uma dessas áreas, muito embora situaçôes semelhantes ocorram nas quatro. Essas formas manifestas e institucionalizadas do movimento humano apresentam papel importante na evolução uma vez que nossa origem genética sempre foi de dinamismo motor em busca do alimento e preservação da espécie. Nossa evolução tornou nosso organismo eficiente em promover deslocamento com a menor demanda energética possível. Essa capacidade econômica faz com que ao repetir uma determinada tarefa motora gastemos menos energia ao longo do tempo. Em contrapartida a vida moderna na era cibernética, passou a demandar do homem contemporâneo menor necessidade motora para as tarefas cotidianas. A cada década temos grande queda na demanda energética diária. Assim podemos dizer que a pandemia da obesidade e apenas uma consequência desses fatos que paradoxalmente chamamos de evolução.

A ciência atual revela dia a dia ajustes na ingestão alimentar e ou suplementação nutricional que aproximam o homem da sua capacidade nominal de realizar esforço. Com base nesse aspecto algumas variáveis merecem atenção quando pensamos em nutrição aplicada a atividade motora. A descoberta 
de qualquer substancia que promova aumento do desempenho humano deve ser considerada segundo dois aspectos resultantes da seguinte pergunta: esta substancia esta garantindo ao indivíduo sua capacidade plena de realização de esforço dentro do seu limite de competência? Se a resposta for sim esta substância ergogênica deve ser encarada como nutriente e sua essencialidade merece atenção da ciência da nutrição; se a resposta for não, ou seja, a substância proporciona desempenho acima do limite de competência do indivíduo, então devemos considerar a mesma como ergogênico não metabólico e sua investigação caberá as autoridades ligadas ao uso de substâncias ilícitas e passíveis de punição perante os comitês esportivos. A preocupação sob o ponto de vista de saúde para o consumo de tais substâncias recaem sobre a população não atleta. Isso porque os atletas são constantemente avaliados pelas respectivas confederações e estão sujeitos a penalidades rigorosas chegando até a bani-los do esporte. Já o individuo comum sofrerá consequências sob o ponto de vista da sua própria saúde sendo que muitas vezes, os sintomas serão percebidos tardiamente quando a preservação da sua integridade pode ser inviável.

Sob esta ótica, o Colégio Americano de Medicina do Esporte juntamente com a Associação de $\mathrm{Nu}$ tricionistas Americanas e Nutricionistas do Canadá um elegante documento onde os principais fatores determinantes do desempenho e ao mesmo tempo preservadores da integridade de saúde dos atletas foi cuidadosamente apresentado (RodrigueZ, Di Marco \& Langley, 2009 ).

\section{Pontos relevantes do conhecimento da Nutrição aplicada a Atividade motora}

\section{Energia}

Os indivíduos ativos precisam consumir energia suficiente durante os períodos de alta demanda energética ou períodos de treinamento de longa duração para manter o peso corporal estável, a saúde e maximizar efeitos do treinamento. Baixo consumo de energia pode resultar em perda de massa muscular, disfunção menstrual, perda ou falha para a manutenção da densidade óssea, aumento do risco de fadiga, lesões, e doença, resultando em processo de recuperação prolongado. Peso corporal e composição não devem ser utilizados como o único critério para a participação nas atividades motoras diária. A observação do peso corporal diária é pratica indesejável sob o risco de estimular práticas que predispõe ao distúrbio alimentar. As fraçôes de gordura corporal ideal dependem do sexo, idade e hereditariedade do indivíduo e pode ser modalidade-específica. As técnicas de avaliação da gordura corporal apresentam variabilidade e limitações inerentes a elas uma vez que todas representam formas indiretas de se determinar o percentual de gordura corporal. Apenas a dissecção de cadáver é a forma direta.

\section{Composição corporal}

As técnicas de avaliação da composição corporal podem ser unicamente indireta (Pesagem hidrostática e pletismografia) e duplamente indireta como dobras corporais, bioimpedância diâmetros e circunferências.

Composição corporal e peso corporal são dois dos muitos fatores que contribuem para o desempenho do exercício em condições ótimas. Juntos, esses dois fatores podem afetar um atleta potencial para o sucesso de um determinado esporte. $\mathrm{O}$ peso corporal pode influenciar a velocidade de um atleta, resistência e potência, enquanto que composição corporal pode afetar a força de um atleta, agilidade, e aparência. Um corpo magro, ou seja, aquele com maior músculo / proporção de gordura, geralmente é vantajoso em esportes onde a velocidade é envolvida. O desempenho atlético não pode ser prevista com precisão baseada apenas no peso corporal e composição, uma vez que muitos fatores podem afetar a composição corporal (Houtkooper, 2000). Algumas modalidades esportivas ditam que os atletas façam alterações no peso corporal assim como na composição que não pode ser melhor para o atleta como as ginastas que precisam de força para suas tarefas e por vezes são cobradas em seu peso. Os atletas que participam em modalidades classificadas pelo peso, tais como "wrestling" ou remo pode ser obrigado a perder ou ganhar peso para se qualificar para uma categoria específica. Os indivíduos que participam de dança, ginástica, patinação artística, ou mergulho, podem ser pressionados para perder peso e gordura corporal para ter um corpo magro, embora o seu peso atual 
para a saúde e o desempenho seja adequado. Com as restriçōes de energia extrema, as perdas da massa muscular e gordura pode influenciar negativamente o desempenho. A Individualização da avaliação da composição corporal, considerando a modalidade esportiva em questão, pode ser vantajosa para a melhoria do desempenho em qualquer circunstancia. Idade, sexo, genética, e as exigências da modalidade são fatores que impactam na composição corporal do atleta. O peso corporal composto por quantidades mínimas de gordura, sem comprometer as respostas fisiológicas, favorecem a competitividade do atleta maximizando seu desempenho. As metodologias e equipamentos para realizar as avaliações de composição corporal devem ser acessíveis e de baixo custo uma vez que serão repetidas inúmeras vezes no ano para o acompanhamento do atleta em suas diversas fases do treinamento. Nem todos os métodos existentes atendem a esses critérios para o praticante. Um exemplo são as metodologias consideradas referências em avaliação de composição corporal como a pesagem hidrostática, pletismografia e DEXA. Esses métodos requerem equipamentos específicos de elevado custo. A utilização dessas técnicas é justificável em modelos científicos que visem avaliar precisamente a alteração na composição corporal dos indivíduos. Para assegurar a saúde do atleta em modalidades classificadas pelo peso corporal é a pesagem destes momentos antes da competição. Assim, aqueles que realizarem a perda de peso para adequação na categoria sofrerão prejuízo no desempenho, pois não terão tempo para a recuperação do estado fisiológico. Com isso a prática de perda de peso rápido seria desestimulada (Artioli, Franchini, Nicastro, Sterkowicz, Solis \& LANCHA JUNIOR, 2010).

Além disso, atletas e treinadores devem saber que existem erros associados com todas as técnicas de avaliação da composição corporal e que elas representam uma estimativa da quantidade de gordura corporal e não uma medida. Em vez disso, uma intervalo de percentagens, ajustados a cada metodologia de composição da gordura corporal deve ser recomendado.

Três níveis técnicos de avaliação são utilizados para avaliar a composição corporal (HEYMSFIELD et al., 2005). A avaliação direta com base na análise de cadáveres, embora não utilizado na prática clínica, é designado como um nível técnico I. Os outros dois níveis técnicos são avaliaçôes indiretas (Nível II) e avaliações duplamente indiretas (Nível III). Hidrodensitometria ou pesagem subaquática, absortometria duplo de energia de raios-X, o mesmo utilizado em densitometria óssea (DXA), e deslocamento de ar pletismografia são técnicas de nível II, e dobras cutâneas medições e análise de impedância bioelétrica (BIA) são técnicas de nível III. Técnicas de Níveis II e III são utilizadas na prática por nutricionistas dedicados a atletas. Pesagem subaquática, considerado o critério padrão de referência, é mais utilizado em estudos científicos. DXA, originalmente desenvolvido para avaliar a mineralização do osso, pode ser usada para a composição corporal (HOUTKOOPER, 2000). Embora DXA seja bastante precisa, rápida e não invasiva, o custo e o acesso aos instrumentos limitam sua utilização na prática. Além disso a avaliação da gordura visceral é prejuicada por esta técnica por utilizar uma análise em duas dimensōes. A pletismografia por ar de deslocamento (BodPod; Vida Medição, Inc., Concord, CA) é também usados para determinar a composição corporal pela densidade do corpo (Heymsfield et al., 2005), e percentual de gordura corporal, calculado usando a equação de SIRI (1956) ou BROzEK (1966). Embora este método ofereça uma avaliação válida e confiável da composição corporal, pode subestimar a gordura corporal em adultos e crianças por $2 \%-3 \%$ (GoING, 2006).

Dois dos mais utilizados métodos de Nível III são as medidas de dobras cutâneas e BIA. Além disso, as medidas de peso, altura, circunferência de pulso e cintura, e medidas de dobras cutâneas são habitualmente utilizadas por nutricionistas para avaliar a composição corporal. Normalmente, são sete locais de dobras cutâneas utilizados, incluindo bíceps, abdominal, coxa, panturrilha medial, subescapular, supraespinhal e tríceps. As técnicas convencionais e definiçóes de cada um destes sitios são fornecidos por HeymSFIELD et al. (2005) e Marfell-Jones, Oldds, Stewart e Carter (2006). As equações de predição por meio de medidas de dobras cutâneas para determinar conteúdo de gordura corporal são numerosas (HEYMSFIELD et al., 2005). Aproximadamente $50 \%-70 \%$ das equações estimam a densidade do corporal e indiretamente projetam o percentual de gordura. Além disso, as diferença populacionais limitam a capacidade de troca das equações de predição e padronização dos sítios de dobras cutâneas, assim como as técnicas variam de autor para autor. Até o compasso de dobras cutâneas é uma fonte de variabilidade (HeYMSFIELD et al., 2005). Apesar de problemas inerentes à medição de dobras cutâneas, esta técnica continua a ser um método de primeira 
escolha, pois é conveniente e barato. Nos EUA o Comité Olímpico (USOC) está usando as técnicas de (MARFELL-Jones et al., 2006) e estão tentando viabilizar esse uso mundialmente. O USOC defende o uso da soma de sete dobras cutâneas ( $\mathrm{mm}$ ) ao invés de percentual de gordura corporal. BIA é baseada no princípio de que um sinal elétrico trafega mais facilmente por meio de tecido magro do que a gordura ou osso (HeYMSFIELD et al., 2005). A massa adiposa é estimada subtraindo-se o BIA que estima a massa magra corporal total (HeYMSFIELD et al., 2005). A bioimpedância elétrica é dependente de vários fatores que podem causar erro na medição e devem ser levados em conta para obter uma estimativa bastante precisa. $\mathrm{O}$ estado de hidratação é o mais importante fator que pode alterar a composição corporal. Sua aplicação é dependente do profissional que aplica a técnica e sua familiarização com a mesma. Ainda assim não é apontada como técnica de primeira escolha.

\section{Macronutrientes}

As recomendações de carboidratos para os atletas vão de 6-10 g. $\mathrm{kg}^{-1}$ de peso corporal-1. Os hidratos de carbono manter a glicemia níveis durante o exercício e repor o glicogênio muscular. A quantidade necessária depende do total energético gasto diário, do tipo de intensidade e duração da atividade, sexo do praticante e condiçóes ambientais. Representa foco de interesse cientifico a ingestão de carboidrato em momentos diferentes da prática da atividade motora assim como a forma química de ingestão como monossacarídeos ou polissacarídeos (RoDriguez, Di Marco \& Langley, 2009).

As recomendaçôes de ingestão de proteína são quase unanimes em permanecer no intervalo 1,2-1,7 g. $\mathrm{kg}^{-1}$. Esta recomendação de ingestão de proteínas geralmente pode ser atendida somente através da dieta, sem a necessidade de proteína ou aminoácidos suplementares. O consumo de energia suficiente atender a necessidade diária é necessário para que a proteína desempenhe sua função principal dê estrutura tecidual ao invés de fonte energética. É motivo de investigação a utilização de tipos diferentes de fontes proteicas e do momento de consumo da mesma com o objetivo de estimular a síntese proteica e tornar a recuperação da atividade mais rápida possível. É também motivo de interesse científico o papel eventual dos aminoácidos sobre mecanismos intracelulares de sinalização da síntese protéica.
A ingestão de lipídios superior a 20\% do total energético não beneficia o desempenho de indivíduos ativos. Os lipídios são fontes de energia, vitaminas e ácidos graxos essenciais, é sua importância na alimentação dos indivíduos ativos saudáveis é ponto de interesse uma vez que a estrutura química dos lipídios pode determinar favorecimento a processos de inflamação ou açôes anti-inflamatórias. As dietas ricas em gordura são desencorajadas pelas pesquisas de uma forma geral.

Indivíduos que restringem o consumo de energia ou uso severo de práticas de perda de peso, eliminando um ou mais grupos de macronutrientes de sua alimentação estão suscetíveis a deficiências de micronutrientes. $\mathrm{O}$ exemplo mais frequente é a restrição ao consumo de alimentos ricos em carboidratos visando a perda de peso (Nattiv, Loucks, Manore, Sanborn, SundgotBorgen \& Warren, 2007). Apenas três nutrientes fornecem energia na nossa alimentação carboidratos, lipídios e proteínas. Assim a restrição de um deles implica naturalmente em elevação dos outros dois. A restrição de carboidratos implica em maior ingestão de gordura como por exemplo a ingestão de queijos, carnes, peixes considerados popularmente fontes proteicas mas que fornecem elevada quantidade de gordura.

A ingestão de todos nutrientes contribui ao equilíbrio de diversos processos metabólicos inclusive a sinalização da interrupção do consumo de alimentos pelos processos de saciedade e saciação. Diversos autores avaliam a restrição de macronutrientes como um fator de estímulo para o consumo compulsivo de alimentos.

Desidratação (déficit hídrico de 2-3\% da massa corporal) diminui o desempenho do exercício, portanto, adequada ingestão de líquidos antes, durante e após o exercício é importante para a saúde e desempenho dentro da capacidade individual. O objetivo de beber é para evitar a desidratação do ocorridos durante o exercício e os indivíduos não devem beber em excesso da taxa de transpiração (PEREIRA-LANCHA, MarqueZi \& LANCHA JunIOR, 2011).

Vegetarianos podem estar em risco de baixa ingestão de energia, proteína, gordura e micronutrientes essenciais, como ferro, cálcio (ZEMEL, 2004), vitamina D (HoLICK, 2004), riboflavina, zinco e vitamina B12. Ainda alvo de interesse científico estudos a médio e longo prazo dessa população são carentes e provavelmente revelarão pontos consideráveis na preservação da saúde desse grupo.

Substâncias específicas como creatina (GUALANO, Novaes, Artioli, Freire, Coelho, Scagliusi, RoGERI, Roschel, UGRINOWITSCH \& LANCHA JunIOR, 
2008), leucina (ZANCHI, NiCASTRO \& LANCHA JUNIOR, 2008), aspartato, apasaragina, $\beta$-Alanina (Artioli, Gualano, Smith, Stout \& Lancha Junior, 2010), betaina, quercitina, glutamina, taurina, cafeina dentre outras, representam motivo de interesse nas investigações de seus efeitos sobre a atividade motora e a resposta a essas investigações as apontarão como nutrientes essenciais ou suplementos ergogênicos avançando sobre o conhecimento das necessidades específicas do ser humano.

\begin{abstract}
Nutrition applied to Motor Activity

Nutrition applied to motor activity is divided in four areas of human movement, namely: sports, physical education, dance, recreation/leisure. This conceptual definition differentiates the target population of nutritional intervention. The human body has always presented the movement as part of their daily activity and selected organisms evolutionarily more economical. In return for the account of demands, social, financial, among other modern life imposed inactivity as the motor pattern of human behavior that together with the genetic default of the economy resulted in the modern diseases such as obesity, diabetes etc. In this way society has institutionalized human movement creating distinct manifestations described above and their specific needs have to be of academic interest / scientific. Nutritionally, the studies focus on energy balance, the need for carbohydrates, proteins, lipids and micronutrients and other biologically active compounds. These studies define these substances under the criteria of essentiality or ergogenic effect than the physiological. The first determines changes in nutritional needs and the second substances deemed illegal. At present much of the scientific community dedicated to nutrition applied to motor activity, directs his calling in an attempt to discover the specific needs caused by the regular practice of motor activity allowing for regular exercise that triggers the same benefits in health maintenance so full in the four areas described above.
\end{abstract}

UnITERMS: Nutrition; Sport; Physical education.

\title{
Agradecimentos
}

Os estudos apresentados neste trabalho foram possíveis pelo apoio FAPESP 2010/11221-0 e 2010/10852-6 e CNPq 308489/2010-14.

\section{Referências}

ARTIOLI, G.G.; FRANCHINI, E.; NICASTRO, H.; STERKOWICZ, S.; SOLIS, M.Y.; LANCHA JUNIOR, A.H. The need of a weight management control program in judo: a proposal based on the successful case of wrestling. Journal of the International Society of Sports Nutrition, Woodland Park, v.7, n.1, p.15, 2010.

ARTIOLI, G.G.; GUALANO, B.; SMITH, A.; STOUT, J.; LANCHA JUNIOR, A.H. Role of $\beta$-alanine supplementation on muscle camosine and exercise performance. Medicine and Science in Sports and Exercise, Hagerstown, v.42, n.6, p.1162-73, 2010.

BROZEK, J. Body composition: models and estimation equations. American Journal of Physical Anthropology, Hoboken, v.24, p.239-46, 1966.

GOING, S. Optimizing techniques for determining body composition. Sports Science Exchange 101, Barrington, v.19, 2006. GUALANO, B.; NOVAES, R.B.; ARTIOLI, G.G.; FREIRE, T.O.; COELHO, D.F.; SCAGLIUSI, F.B.; ROGERI, P.S.; ROSCHEL, H.; UGRINOWITSCH, C.; LANCHA JUNIOR, A.H. Effects of creatine supplementation on glucose tolerance and insulin sensitivity in sedentary healthy males undergoing aerobic training. Amino Acids, Wien, v.34, p.245-50, 2008.

50 • Rev. bras. Educ. Fís. Esporte, São Paulo, v.25, p.45-51, dez. 2011 N. esp. 
HEYMSFIELD, S.; LOHMAN, T.; WANG, Z.; GOING, S. Human body composition. 2nd ed. Champaign: Human Kinetics, 2005.

HOLICK, M.F. Vitamin D: importance in the prevention of cancers, type 1 diabetes,heart disease, and osteoporosis. American Journal of Clinical Nutrition, Bethesda, v.79, p.362-71, 2004.

HOUTKOOPER, L. Body composition. In: MANORE, M.; THOMPSON, J. (Eds.). Sport nutrition for health and performance. Champaign: Human Kinetics, 2000.

LANCHA JUNIOR, A.H.; RIANI, L. Conceitos. In: LANCHA JUNIOR, A.H.; PEREIRA-LANCHA, L.O. Nutrição aplicada à atividade motora. 3.ed. São Paulo: Atheneu, 2011.

MARFELL-JONES, M.; OLDS, T.; STEWART, A.; CARTER, L. International standards for anthropometric assessment. Potchefstroom: International Society for the Advancement of Kinanthropometry, 2006.

NATTIV, A.; LOUCKS, A.B.; MANORE, M.M.; SANBORN, C.F.; SUNDGOT-BORGEN, J.; WARREN, M.P.; American College of Sports Medicine. American College of Sports Medicine position stand. The female athlete triad. Medicine and Science in Sports and Exercise, Hagerstown, v.39, n.10, p.1867-82, 2007.

PEREIRA-LANCHA, L.O.; MARQUEZI, M.L.; LANCHA JUNIOR, A.H. Hidratação. In: LANCHA JUNIOR, A.H.; PEREIRA-LANCHA, L.O. Nutrição aplicada à atividade motora. 3.ed. São Paulo: Atheneu, 2011.

RODRIGUEZ, N.R.; DI MARCO, N.M.; LANGLEY, S. American College of Sports Medicine position stand. Nutrition and athletic performance. American Dietetic Association, Dietitians of Canada, American College of Sports Medicine. Medicine and Science in Sports and Exercise, Hagerstown, v.41, n.3, p.709-31, 2009.

SIRI, W. Gross composition of the body. In: LAWRENCE, J.; CORNELIUS, A. (Eds.). Advances in biological and medical physics. New York: Academic Press, 1956.

ZANCHI, N.E.; NICASTRO, H.; LANCHA JUNIOR, A,H. Potencial antiproteolytic effects of L-leucine: observaions of in vitro and in vivo studies. Nutrition \& Metabolism, London, v.5, p.20, 2008.

ZEMEL, M.B. Role of calcium and dairy products in energy partitioning and weight management. American Journal of Clinical Nutrition, Bethesda, v.79, p.907S-12S, 2004. Supplement.

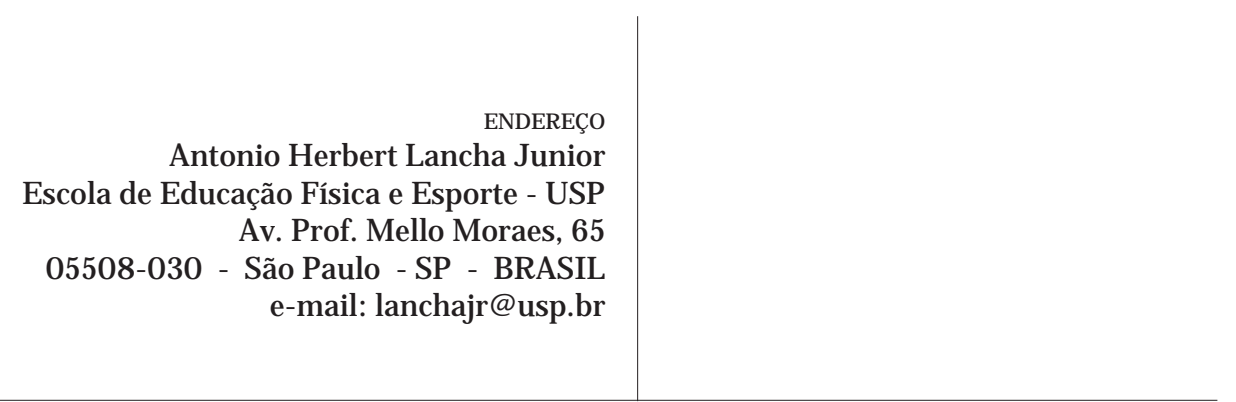

